Published in final edited form as:

Biochemistry. 2015 December 29; 54(51): 7449-7456. doi:10.1021/acs.biochem.5b01078.

\title{
Backbone Flexibility Influences Nucleotide Incorporation by Human Translesion DNA Polymerase $\eta$ Opposite Intrastrand Cross-linked DNA
}

\author{
Derek K. O’Flaherty $\$$, F. Peter Guengerich ${ }^{\ddagger}$, Martin Egli ${ }^{\ddagger},{ }^{\star}$, and Christopher J. Wilds ${ }^{\S,}{ }^{*}$ \\ $\S$ Department of Chemistry and Biochemistry, Concordia University, 7141 Sherbrooke St. West, \\ Montréal, Québec (Canada) H4B 1R6 \\ ‡Department of Biochemistry and Center in Molecular Toxicology, Vanderbilt University School of \\ Medicine, Nashville, Tennessee (USA) 37232-0146
}

\begin{abstract}
Intrastrand cross-links ( $\mathrm{IaCL}$ ) connecting two purine nucleobases in DNA pose a challenge to high fidelity replication in the cell. Various repair pathways or polymerase bypass can cope with these lesions. The influence of the phosphodiester linkage between two neighbouring $2^{\prime}$-deoxyguanosine (dG) residues attached through the $O^{6}$-atoms by an alkylene linker on bypass with human DNA polymerase $\eta(\mathrm{hPol} \eta)$ was explored in vitro. Steady-state kinetics and mass spectrometry analysis of products from nucleotide incorporation revealed that although hPol $\eta$ is capable of bypassing the $3^{\prime}$-dG in a mostly error-free fashion, significant misinsertion was observed for the $5^{\prime}$-dG of the IaCL containing a butylene or heptylene linker. The lack of the phosphodiester linkage triggered a significant increase in frameshift adduct formation across the $5^{\prime}-\mathrm{dG}$ by hPol $\eta$, in comparison to the $5^{\prime}$-dG of IaCL DNA containing the phosphodiester group.
\end{abstract}

\section{Keywords}

Intrastrand cross-linked DNA; DNA damage; DNA polymerase; enzyme kinetics

\section{INTRODUCTION}

Chemotherapeutic agents such as the platinum-containing drugs used in the treatment of cancer exert their therapeutic effect mainly via the formation of cytotoxic DNA damage. The lesions that these agents produce have been identified primarily as intrastrand cross-links (IaCL) between the N7-atoms of purines with the distribution of these IaCL determined to

\footnotetext{
“Corresponding Author: ; Email: chris.wilds@concordia.ca, ; Email: martin.egli@ vanderbilt.edu Author Contributions

All authors conceived and designed the experiments. DKO performed all the experiments and analyzed the data. All authors interpreted the results and wrote the manuscript.

Notes

The authors declare no competing financial interests.

Supporting Information. Example of IaCL DNA mentioned in the manuscript. Characterization by ESI-MS of the modified oligomers. LC-MS analysis of most abundant full-length extension products opposite modifications in DNA template by hPol $\eta$ in the presence of all four dNTPs. This material is available free of charge via the Internet at http://pubs.acs.org.
} 
be $65 \% 1,2(\mathrm{GpG}), 25 \% 1,2(\mathrm{ApG})$, and $5-10 \% 1,3(\mathrm{GpTpG}) .{ }^{1 \_9}$ In addition, minor formation of other products including interstrand cross-links (ICL), mono-adducts and DNA-protein cross-links occurs. ${ }^{9}$ The presence of these adducts on the DNA scaffold impedes vital cellular processes such as DNA replication and transcription ultimately leading to cell death. Drugs used in cancer regimens, other than platinum-containing agents, such as mechlorethamine, ${ }^{10},{ }^{11}$ mitomycin $\mathrm{C}^{12,13}$ and busulfan ${ }^{14}$ have also been shown to introduce IaCL in DNA, in particular between adjacent purine nucleobases. Using drugs that act directly on DNA to treat cancer, have intrinsic and acquired drug resistance as a major limitation, which is mediated by cellular response processes like DNA repair and translesion DNA synthesis (TLS).

The four TLS DNA polymerases identified in humans are Pol $\eta$, Pol $\kappa$, Pol $l$, and Rev1. The most widely studied of these is Pol $\eta$ given its crucial involvement in bypassing UV-induced intrastrand cross-linked DNA lesions. Disruption in the proper function of the $P O L H$ gene leads to xeroderma pigmentosum variant (XPV), a condition characterized by hypersensitivity to UV-irradiation and an increased incidence of skin cancer. ${ }^{15}$ As suspected, $P O L H$ knockout mice demonstrated heightened incidences of skin cancer compared to the control group when exposed to UV-irradiation. ${ }^{16} \mathrm{XPV}$ cell extracts displayed replication inhibition of plasmid DNA containing a single (6-4) pyrimidone photoproduct lesion. ${ }^{17}$ Moreover, human cells deficient in Pol $\eta$ revealed greater cell death events when treated with platinum-based chemotherapeutic agents. ${ }^{18}{ }^{21}$ Exposure of DNA to $\gamma$-irradiation leads to the formation of a mixture of the IaCL lesions G[8,5]C and G[8,5]T, among others, formed via a radical mechanism. ${ }^{22}$ Their bypass by yeast and/or human Pol $\eta$ demonstrated reduced fidelity and processivity, in particular across the 2 -deoxyguanosine portion of the lesion. $^{23} 25$ Accounts of Pol $\eta$ bypass are numerous and the search for other biologically relevant DNA damage, or mimics thereof, is ongoing.

DNA alkylating agents such as $N$-nitroso- $N$-methylurea readily modify the N7-atom of dG and, to a lesser extent, the $O^{6}$-position. Lesions at the $O^{6}$-atom of dG have also been detected after exposure to the methylating and chloroethylating chemotherapeutic drugs temozolimide and carmustine. Endogenous formation of $O^{6}$-MedG by $S$ adenosylmethionine is estimated at 10-30 damage events per cell per day. ${ }^{26}$ The introduction of covalent appendages at the N7-atom of a purine nucleotide in DNA reduces chemical stability, leading to modifications such as the introduction of abasic sites and/or formation of formamidopyrimidine lesions. Alkyl modifications introduced at the $O^{6}$ position of dG are chemically stable and if left uncorrected, lead to stalls or significant G:C to A:T transitions by DNA polymerases including those of the Y-family. ${ }^{27} 32$ The importance of this site of alkylation has been demonstrated by in vivo studies which revealed the direct link between $O^{6}$-MedG and carcinogenesis. ${ }^{33}$ The disruption of high fidelity DNA polymerase activity in the presence of $O^{6}$-MedG and other $O^{6}$-alkyldG lesions has been attributed to disruption of Watson-Crick base pairing.

Much less is known about the bypass of IaCL containing more flexible lesions such as an alkylene linker, in comparison to more rigid systems including platinum-based IaCL DNA. In the present study, we investigated whether Pol $\eta$ was capable of efficiently bypassing an $O^{6}$-dG-alkylene- $O^{6}$-dG IaCL containing butylene or heptylene tethers (Figure $1 \mathrm{~A}$ ). Our 
phosphoramidite synthetic strategy used to generate such IaCL DNA probes ${ }^{34}$ allowed us to engineer identical sets of adducted DNA lacking the phosphodiester linkage at the crosslinked site (Figure 1B), which were also evaluated. The absence of the phosphodiester linkage presumably confers increased flexibility to the IaCL DNA. This study set out to (i) investigate the ability of $\mathrm{hPol} \eta$ to bypass a malleable IaCL lesion that can disrupt the fidelity of Watson-Crick base pairing ( $O^{6}$-position of $\mathrm{dG}$ as opposed to the N7-position for instance). The second objective was to (ii) investigate the effect of IaCL linker size (butylene vs. heptylene) on the processivity of hPol $\eta$. Sizes of the IaCL linkers were inspired from DNA adducts formed from exposure to alkyl sulphones Busulfan and Hepsulfam, known to generate butylene and heptylene DNA adducts, respectively (ref). It should be noted, however, that a specific IaCL connected at $O^{6}$-atoms of $5^{\prime}$-d(GG) sequences has not been identified to date. Our model IaCL DNA are utilized as chemically stable probes that can be prepared in scales and purity amenable to biochemical studies. The final objective of the study was to (iii) investigate the effect of phosphodiester linkage deletion on IaCL DNA bypass by $\mathrm{hPol} \eta$, in comparison to those containing this functional group.

\section{MATERIALS AND METHODS}

\section{Chemical Synthesis and Characterization of Modified DNA Oligonucleotides}

The chemical synthesis of the dimer phosphoramidites required to synthesize GG4 and GG7 have been described in previously published work. ${ }^{34}$ Those required for GpG4, and GpG7 are described in a manuscript submitted for review with synthesizes adapted from previously described procedures. ${ }^{35}$ The cross-linked duplexes, whose sequences are $5^{\prime}$-AC XX CT CAC ACT (where $\mathbf{X X}$ denotes the cross linked dGG residues or dGG for the unmodified control), were assembled on an Applied Biosystems Model 3400 synthesizer on a $1.5 \mu \mathrm{mol}$ scale using standard $\beta$-cyanoethylphosphoramidite chemistry supplied by the manufacturer with slight modifications to coupling times. The nucleoside phosphoramidites containing fast deprotecting groups were prepared in anhydrous $\mathrm{CH}_{3} \mathrm{CN}$ at a concentration of $0.1 \mathrm{M}$ for the $3^{\prime}$ - $O$-deoxyphosphoramidites, and $0.15 \mathrm{M}$ for the cross-linked $3^{\prime}-O$ deoxyphosphoramidite. Oligomer sequence assembly was carried out according to published procedures by our group.

Protecting groups and cleavage from the solid support was carried out by treatment with aqueous $(28 \% \mathrm{v} / \mathrm{v}) \mathrm{NH}_{4} \mathrm{OH}$ in $\mathrm{C}_{2} \mathrm{H}_{5} \mathrm{OH}\left(1 \mathrm{~mL}\right.$ of a $3: 1 \mathrm{v} / \mathrm{v}$ solution) for $4 \mathrm{~h}$ at $55^{\circ} \mathrm{C}$ in 2 $\mathrm{mL}$ screw cap microfuge tubes fitted with Teflon lined caps for GG4 and GG7. Modified oligomers GpG4 and GpG7 were first deprotected for $16 \mathrm{~h}$ in aqueous (28\% v/v) ammonium hydroxide at room temperature with gentle rocking, followed by an additional 4 $\mathrm{h}$ at $55{ }^{\circ} \mathrm{C}$. Crude oligomers were transferred and the solvent removed using a Savant SC110A SpeedVac Concentrator (Thermo) followed by purification by strong-anion exchange HPLC with a Dionex DNAPAC PA-100 column $(0.4 \mathrm{~cm} \times 25 \mathrm{~cm})$ purchased from Dionex, Sunnyvale, CA using a linear gradient of $0-52 \%$ buffer B (v/v) over 24 min (buffer A: $100 \mathrm{mM}$ Tris $\mathrm{HCl}, \mathrm{pH} 7.5,10 \% \mathrm{MeCN}$ and buffer B: $100 \mathrm{mMTris} \mathrm{HCl}, \mathrm{pH} 7.5,10 \%$ $\mathrm{CH}_{3} \mathrm{CN}, 1 \mathrm{M} \mathrm{NaCl}$ ) at $55^{\circ} \mathrm{C}$. The columns were monitored at $260 \mathrm{~nm}$ for analytical runs or $280 \mathrm{~nm}$ for preparative runs. The purified oligomers were desalted using C-18 SEP PAK cartridges (Waters) as previously described. ${ }^{36}$ ESI mass spectra for oligonucleotides were 
obtained at the Concordia University Centre for Biological Applications of Mass

Spectrometry using a Micromass Qtof2 mass spectrometer (Waters) equipped with a nanospray ion source. The mass spectrometer was operated in full scan, negative ion detection mode. The molecular mass of the modified oligomers were identifed by ESI-MS and the measured values were in agreement with the expected masses (see Supporting Figures S2-S4 for MS spectra).

\section{Steady-state Kinetics}

All primer extension assays were carried out using template strand with sequence $5^{\prime}$-AC XX CT CAC ACT (where $\mathbf{X X}$ denotes the cross linked dGG residues or dGG for the unmodified control) and a fluorescently labelled primer $5^{\prime}$-(FAM)TAG TG $U$ GAG (where $U$ is $2^{\prime}$ deoxyuridine). Steady-state kinetic experiments were conducted as previously described. ${ }^{37} 40$ Briefly, assays were generally performed at $37^{\circ} \mathrm{C}$ in $40 \mathrm{mM}$ Tris-HCl buffer (pH 7.5) containing $100 \mathrm{mM} \mathrm{KCl}, 5 \%$ glycerol (v/v), $10 \mathrm{mM}$ dithiothreitol (DTT), $5 \mathrm{mM}$ $\mathrm{MgCl}_{2}$ and $100 \mu \mathrm{g} \mathrm{mL}^{-1}$ bovine serum albumin (BSA). The 5'-labelled 6carboxyfluorescein (FAM) primer-template (9-/13-mer) duplex $(5 \mu \mathrm{M})$ was extended using 1.9 to $500 \mathrm{nM}$ concentrations of $\mathrm{hPol} \eta$ in the presence of various concentrations of a single dNTP ( 0 to $1 \mathrm{mM}$, at 7-10 different dNTP concentrations) at $37^{\circ} \mathrm{C}$ for $5-20 \mathrm{~min}$. Reactions were quenched using a solution containing $20 \mathrm{mM}$ EDTA ( $\mathrm{pH} 8.0$ ), 95\% formamide (v/v), bromphenol blue, and xylene cyanol dyes. Substrates and products were resolved on $18 \%$ (w/v) polyacrylamide electrophoresis gels containing 7.5 M urea. Gels were monitored by a Typhoon Scanner (GE Healthcare) and analyzed by fluorescence intensity using ImageJ software (National Institutes of Health). The values of $k_{\text {cat }}$ and $K_{\mathrm{m}}$ were estimated by nonlinear regression analysis (hyperbolic fit) with the program Graphpad Prism (La Jolla, CA).

\section{LC-MS/MS Analysis of Fully Extended Products}

All primer extension assays were carried out using template strand with sequence $5^{\prime}$-AC XX CT CAC ACT (where $\mathbf{X X}$ denotes the cross linked dGG residues or dGG for the unmodified control) and fluorescently labelled primer 5'-(FAM)TAG TG $U$ GAG (where $U$ is $2^{\prime}$ deoxyuridine). DNA Primers were extended in the presence of all four dNTP followed by analysis via mass spectrometry. Primer sequences contained a 2'-deoxyuridine (U) in order to easily cleave products to a shorter oligonucleotide (by treatment with uracil DNA glycosylase followed by hot piperidine), which was subsequently analyzed by an LCMS/MS method (ion-trap mass spectrometer), as previously described. ${ }^{37,38,41}{ }^{43}$ DNA primer extension was accomplished by combining hPol $\eta(95 \mathrm{pmol}, 0.95 \mu \mathrm{M}$ for unmodified duplexes and $340 \mathrm{pmol}, 0.95 \mu \mathrm{M}$ for IaCL-containing duplexes) with template-primer duplex $(2 \mathrm{nmol}, 10 \mu \mathrm{M})$ and a mixture of $1 \mathrm{mM}$ each of dATP, dCTP, dGTP, and dTTP at $37^{\circ} \mathrm{C}$ for $0.5-1.5 \mathrm{~h}$ in $50 \mathrm{mM}$ Tris- $\mathrm{HCl}$ buffer ( $\mathrm{pH} 7.5$ ), $50 \mathrm{mM} \mathrm{NaCl}, 5 \mathrm{mM}$ DTT, $5 \mathrm{mM}$ $\mathrm{MgCl}_{2}$ and $50 \mu \mathrm{g} / \mathrm{ml}$ bovine serum albumin (BSA). The reactions were terminated by spin column separations (Micro Bio-Spin ${ }^{\mathrm{TM}} 6$ Columns from BIO-RAD) to extract the dNTPs and $\mathrm{Mg}^{2+}$. The extent of the extension was monitored by electrophoresis/fluorography prior to LC-MS analysis (Supporting Figure 6). The resulting product was then treated with 25 units of uracil DNA glycosylase and $0.25 \mathrm{M}$ piperidine. ${ }^{37,38,41 \_43}$ To identify the products, the resulting reactions were analyzed by LC-MS/MS using an Acquity UPLC system (Waters) interfaced to a Thermo-Finnigan LTQ mass spectrometer (Thermo Scientific, San Jose, CA) 
equipped with a negative ion electrospray source. Chromatographic separation was carried out with an Acquity UPLC BEH octadecylsilane (C18) column $(2.1 \times 100 \mathrm{~mm}, 1.7 \mu \mathrm{m})$. The LC solvent system was as follows: Mobile phase A, $10 \mathrm{mM} \mathrm{CH}_{3} \mathrm{CO}_{2} \mathrm{NH}_{4}$ in $98 \% \mathrm{H}_{2} \mathrm{O}$; mobile phase $\mathrm{B}, 10 \mathrm{mM} \mathrm{CH}_{3} \mathrm{CO}_{2} \mathrm{NH}_{4}$ in $90 \% \mathrm{CH}_{3} \mathrm{CN}(\mathrm{v} / \mathrm{v})$. The following gradient (v/v) was used with a flow rate of $300 \mu \mathrm{L} \mathrm{min}^{-1}$ at a temperature of $50{ }^{\circ} \mathrm{C}$ : Linear gradient from 0-3\% B (v/v) in $3 \mathrm{~min}$, followed by a linear increase to $20 \% \mathrm{~B}(\mathrm{v} / \mathrm{v})$ from 3-5 min, then 20$100 \% \mathrm{~B}(\mathrm{v} / \mathrm{v})$ from 5-6 min which was held for $2 \mathrm{~min}$. The column was re-equilibrated for 3 min with $0 \% \mathrm{~B}(\mathrm{v} / \mathrm{v})$ for subsequent analysis. Mass spectrometry conditions were as follows: Source voltage, $4 \mathrm{kV}$; source current $100 \mu \mathrm{A}$; capillary voltage,- $49 \mathrm{~V}$; capillary temperature, $350^{\circ} \mathrm{C}$; tube lens voltage, $-90 \mathrm{~V}$. Product ion spectra were recorded over the range $\mathrm{m} / \mathrm{z}$ 300-2000 and the most abundant species (-2 charge) was used for collisioninduced dissociation (CID) analysis. The calculation for the oligonucleotide sequence CID fragmentation was carried out using Mongo Oligo Mass Calculator v2.06 from The RNA Institute (College of Arts and Science, University at Albany State University of New York). The relative yields of various products were calculated based on the peak areas of extracted ion chromatograms from LC-MS analyses. The sum of the peak areas was used for multicharged species.

\section{RESULTS AND DISCUSSION}

DNA duplexes containing an $O^{6}$-dG-alkylene- $O^{6}-\mathrm{dG}$ IaCL and lacking a phosphodiester linkage between the tethered nucleotides have been shown to be substrates of the human DNA repair protein $O^{6}$-alkylguanine DNA alkyltransferase (hAGT), which repairs the linkage restoring $\mathrm{dG}^{34}$ In this process, the first reaction with AGT forms two products, a DNA-protein cross-linked (DPC) species and a repaired oligonucleotide. The DPC is a substrate for a second repair event which releases the second unmodified (repaired) DNA fragment. Butylene linked IaCL were observed to be resistant to the action of hAGT using extended reaction times (8h) and five molar equivalents of the protein. However, the heptylene linked IaCL DNA analogues were almost completely consumed under these conditions. $^{34}$

Bypass studies with hPol $\eta$ have revealed its ability to process a wide variety of DNA modified at the nucleobase including $O^{6}$-alkyl-dG adducts ${ }^{28}, 1, N^{2}$-etheno- $2^{\prime}$ deoxyguanine $^{44}$, and 8-oxo-7,8-dihydro-2'-deoxyguanosine ${ }^{37}$. Bulkier forms of DNA damage including intrastrand lesions induced by cisplatin ${ }^{45,46}$ and UV radiation ${ }^{15,47}$, such as $\mathrm{dG}[8,5-\mathrm{Me}] \mathrm{dT} \mathrm{IaCL}^{23}$, have also been the subject of bypass studies with hPol $\eta$. Bisalkylating drugs such as busulfan can introduce IaCL that may have increased flexibility at the modified site relative to the bulkier lesions described above. Given the versatility of hPol $\eta$ to bypass a variety of lesions by TLS, we decided to explore the influence of flexibility at the modified site with $O^{6}$-dG-alkylene- $O^{6}$-dG IaCL containing either a butylene or heptylene linkage between the bases. To further probe the influence of flexibility, template strands lacking and containing a phosphodiester linkage between the linked nucleobases have been prepared (see Supporting Information Figures S2-S5 for ESI-MS of the template strands). The template strands employed in this study were designed based on cisplatinadducted DNA sequences studied by Zhao and coworkers. ${ }^{46}$ 
Steady-state kinetic evaluations of nucleotide incorporations opposite IaCL lacking a phosphodiester linkage between the attached nucleobases (GG4 and GG7, where 4 and 7 are butylene and heptylene linked), containing a phosphodiester linkage (GpG4 and GpG7) and unmodified GG were carried out with the catalytic core construct of hPol $\eta$ (amino acids 1-432). The primer extension assay is described pictorially in Figure 2. In all cases, the IaCL modification blocked DNA synthesis by hPol $\eta$ relative to the unmodified control. Incorporation of the correct dCMP nucleotide by hPol $\eta$ opposite the first $3^{\prime}$-alkylated dG of GG4, GG7, GpG4, and GpG7 was reduced by approximately 320-, 280-, 320-, and 320fold, respectively, relative to GG (see Figure 3 and Supporting Information Table S1). These results differ significantly from the cisplatin-adducted GG templates where a 1.2 fold decrease was observed, ${ }^{46}$ which suggests that modification of the Watson-Crick hydrogenbonding face poses a challenge to the incorporation efficiency of hPol $\eta$. These results correlate with studies of hPol $\eta$ bypass with an $O^{6}$-MedG-containing template, which demonstrated a significant decrease (10-fold) in efficiency for incorporation of the correct dCMP nucleotide. ${ }^{28}$ The reduced efficiency of incorporation may be attributed to hindrance due to the presence of the alkylene tether. Bulkier lesions at the $O^{6}$-atom such as the benzyl and 4-oxo-4-(3-pyridyl)butyl] lesion have been shown to reduce incorporation efficiency by a factor of $\sim 65$ and 250 , respectively, for hPol $\eta{ }^{28}$ The alkylene linkage to the subsequent nucleotide could also contribute to the reduced relative kinetic efficiencies observed. Significant misinsertions by Saccharomyces cerevisiae Pol $\eta$ have also been observed across the $\mathrm{dG}$ nucleotide of the $\mathrm{dG}[8,5-\mathrm{Me}] \mathrm{dT}$ IaCL, whereas hPol $\eta$ incorporated the correct dNMPs across both nucleotides of this IaCL. ${ }^{23,},{ }^{25},{ }^{48}$ Steady state data for our IaCL DNA showed a preference for dCMP insertion across the first $3^{\prime}$-dG alkylated residue. However, an overall decrease in selectivity ( $f$ coefficients are shown in Figure 3 and supplied in Supporting Table S1) was observed, particularly for the GG4 and GG7 templates. Relative insertions ( $f$ coefficients) of dAMP, dGMP and dTTP increased to $0.37,0.34$, and 0.24 for GG4 and 0.40, 0.23, and 0.47 for GG7 compared to $0.001,0.001$, and 0.003 for GG, respectively. Lower $f$ coefficients were generally observed for GpG4 and GpG7 compared to GG4 and GG7, respectively, suggesting the added flexibility inherent to GG4 and GG7 posed an issue for hPol $\eta$ fidelity. It was interesting to observe an increased efficiency and selectivity for the butylene linkers (GG4 and GpG4) in comparison to heptylene linkers (GG7 and GpG7), corroborating that increased steric bulk hinders processivity by hPol $\eta$. It was noted that in all cases, except GG4, dTMP was incorporated most-efficiently out of the misinsertions across the $3^{\prime}$-dG position.

Post-insertion extension across the second alkylene linked site ( $\left.5^{\prime}-\mathrm{dG}\right)$ using primers that contain $\mathrm{dC}$ or $\mathrm{dT}$ directly across from the $3^{\prime}-\mathrm{dG}$ of the IaCL containing templates, as well as the unmodified GG, was studied. The results are summarized in Figure 3 and tabulated in Supporting Tables S2 and S3. Extension across the second $O^{6}$-alkylene linked dG was generally slower compared to the first regardless of the identity of the additional nucleotide (dC or $\mathrm{dT}$ ) in the primer. An interesting feature for the GG4 and GG7 templates was the apparent proficient misinsertion of dGTP across the $O^{6}$-alkylated $5^{\prime}$-dG residue. It was suspected that frameshift formation had occurred, which was confirmed by full extension analysis by LC-MS/MS, given that $\mathrm{dC}$ is the subsequent nucleotide in the template strand. dNTPs other than dGTP were not efficiently incorporated in the case of GG4 and GG7. The 
post-extension profiles for GpG4 and GpG7 displayed a clear decrease in selectivity compared to the insertion across the first ( $\left.3^{\prime}\right) O^{6}$-alkylated-dG, with an almost complete loss in dNTP preference for GpG7. To be noted is the preference of dTTP over dCTP for GpG4 and GpG7. The $3^{\prime}$-end $O^{6}$-alkylated-dG $\bullet$ dT mismatch significantly lowered incorporation efficiency across the subsequent $O^{6}$-alkylated-dG for all IaCL DNA except for insertion of dCMP in the case of GpG4. The steady state results showed an overall reduction in incorporation efficiency and fidelity, particularly across the $5^{\prime}-\mathrm{dG}$ of the IaCL. This occurrence is not observed for hPol $\eta$ bypass of platinum-based dGG adducts nor the $\mathrm{dG}[8,5-\mathrm{Me}] \mathrm{dT}$ adduct. ${ }^{49}$ The reduction of incorporation efficiency and fidelity across the $5^{\prime}$ $\mathrm{dG}$ may be the result of the alkylene linker adopting an orientation that disrupts efficient DNA primer extension.

Analysis of single insertions by a DNA polymerase is useful for kinetic analysis in terms of identifying the extent of blockage by a given modification in a quantitative manner. However, this may not reflect insertion profiles in the presence of all four dNTPs as well as extension past the damaged sites. Fidelity of hPol $\eta$ and processivity across and past the damage site was assessed via analysis of extended products by LC-MS/MS, using a reported methodology. ${ }^{28,37,41}{ }^{43}$ This method provides insight regarding hPol $\eta$ 's preference to incorporate dNTP across the IaCL site (Supporting information Tables S4 and S5). The PAGE analysis of fully extended products (Figure 4) revealed blockage of hPol $\eta$ after insertion across the GG4 and GG7 IaCL modification (e.g. extension was stalled after incorporation of the second dNTP). Similar results were observed for replication across and past DNA templates containing a single abasic site insert and may represent slippage of hPol $\eta$ during replication. ${ }^{38}$ Our study suggests that hPol $\eta$ bypass requires an intact phosphodiester DNA backbone for processivity, particularly for IaCL. In contrast, hPol $\eta$ replicated past the IaCL of GpG4 and GpG7 to reach an accumulation of the fully extended $(13 \mathrm{nt})$ and over-extended (14 nt) product (Figure 4). hPol $\eta$ exhibited similar bypass extension profiles for other $O^{6}$-alkyl-dG lesions, which suggested that the IaCL containing a phosphodiester linkage may be processed as two adjacent mono-adducted dG inserts.

Insertion profiles opposite the $3^{\prime}-\mathrm{dG}$ and $5^{\prime}-\mathrm{dG}$ of GG4, GG7, GpG4 and GpG7 and the unmodified control are shown in Figure 5. Insertion of dCMP across the $3^{\prime}$-end of the $O^{6}$ dG-alkylene- $O^{6}$-dG was preferred by hPol $\eta$ in all cases, which was in agreement with the steady state kinetic data. Although the DNA replication machinery has been shown to misinsert dTMP opposite $O^{6}-\mathrm{MedG}^{28} 31$, only a slight increase (3 - 12\%) in dTMP insertion by hPol $\eta$ across the $3^{\prime}-\mathrm{dG}$ relative to the unmodified control was observed. Interestingly, the MS-based analysis revealed that dAMP incorporation occurs at levels of 24\% and 14\% for GG4 and GG7, respectively, and in only negligible amounts for GpG4, GpG7 and the unmodified control. Similar erroneous dAMP insertions by hPol $\eta$ have been observed opposite abasic sites (also known as the "purine-rule") in addition to frameshift adduct formation. ${ }^{38,50 \_52}$ Minor products $(<3 \%)$ corresponded to frameshift adduct formation opposite $3^{\prime}$-dG lesions.

Nucleotide insertion profiles for the $5^{\prime}$-end of the dG lesion by hPol $\eta$ revealed proclivity for the frameshift adduct formation in the case of GG4 and GG7, whereas a pyrimidine insertion was preferred for GpG4 and GpG7. These results were in agreement with the 
steady state kinetic evaluation of dGMP insertion across the $5^{\prime}$-end of the dG lesion of GG4 and GG7. We suspect that the added flexibility incurred from the lack of a phosphodiester linkage is responsible for large discrepancies observed between the IaCL DNA. hPol $\eta$ incorporated little to no purines opposite the $5^{\prime}$-end of the dG lesion in GpG4 and GpG7, but levels of $10 \%$ and $17 \%$ for dAMP and dGMP, respectively, were measured for GG4. Only $7 \%$ dAMP was detected for extension across the $O^{6}$-alkylated $5^{\prime}$-dG in GG7, with no dGMP insertion detected. Misinsertion of dTMP was preferred by hPol $\eta$ in the case of GpG4 and GpG7 (53\% and 74\%, respectively). Correct insertion of dCMP accounted for $47 \%$ and $24 \%$ opposite the $O^{6}$-alkylated $5^{\prime}$-dG in GpG4 and GpG7, respectively, and only a minor dCMP insertion was observed for GG4 and GG7 (11\% and 1\%, respectively).

\title{
CONCLUSION
}

In conclusion, both the steady state primer extension assays and LC-MS analysis of fully extended product demonstrated that extension opposite the $5^{\prime}$-end of the $O^{6}$-dG-alkylene$O^{6}$-dG was more problematic compared to the $3^{\prime}$-end. An increase in frameshift adduct formation by hPol $\eta$ was observed at $5^{\prime}$-dG of IaCL DNA lacking the phosphodiester linkage, compared to those IaCL containing this functional group. To be noted was the decrease in selectivity by hPol $\eta$ for longer heptylene IaCL versus the butylene analogue, regardless of the presence of the phosphodiester group at the cross-linked site. Structural insights on the ternary complexes (DNA, hPol $\eta$ and incoming nucleoside triphosphate) by $\mathrm{X}$-ray crystallography are underway and will shed light on the error-free bypass and errorprone behavior of hPol $\eta$ towards these IaCL.

\section{Supplementary Material}

Refer to Web version on PubMed Central for supplementary material.

\section{Acknowledgments}

\begin{abstract}
Funding Sources
This work was supported by grants from the Natural Sciences and Engineering Research Council (NSERC) of Canada and Canada Research Chair Program (C.J.W.) as well as the US National Institutes of Health (R01 ES010375 to F.P.G. and M.E. and P01 CA160032 to M.E.). D.K.O. was a recipient of a postgraduate fellowship (CGS-D) and the Michael Smith Foreign Study Supplement Program from NSERC.
\end{abstract}

The authors are grateful to Dr. Amritraj Patra and Dr. Anne Noronha for helpful discussions.

\section{ABBREVIATIONS}

$\begin{array}{ll}\mathbf{d G} & 2 \text { '-deoxyguanosine } \\ \mathbf{3}^{\prime} \text {-dG } & \mathrm{dG} \text { residue at the } 3^{\prime} \text {-end of the } \mathrm{IaCL} \\ \mathbf{5}^{\prime} \text {-dG } & \mathrm{dG} \text { residue at the } 3^{\prime} \text {-end of the } \mathrm{IaCL} \\ \text { TLS } & \text { translesion synthesis } \\ \text { Pol } & \text { DNA polymerase }\end{array}$


XPV

UV

$O^{6}$-MedG

ESI-MS

PAGE

hPol $\eta$

CID

dNTP xeroderma pigmentosum variant

ultraviolet

O6-methyl-2'-deoxyguanosine

electrospray ionization mass spectrometry

polyacrylamide gel electrophoresis

human DNA polymerase $\eta$

collision-induced dissociation

2'-deoxynucleoside triphosphate

\section{References}

1. Bruhn SL, Toney JH, Lippard SJ. Biological Processing of DNA Modified by Platinum Compounds. Prog Inorg Chem. 1990; 38:477-516.

2. Eastman A. Characterization of the Adducts Produced in DNA by cis-Diamminedichloroplatinum (II) and cis-Dichloro(ethylenediamine)platinum(II). Biochemistry. 1983; 22:3927-3933. [PubMed: 6225458]

3. Eastman A. Interstrand Cross-Links and Sequence Specificity in the Reaction of cisDichloro(ethylenediamine)platinum(II) with DNA. Biochemistry. 1985; 24:5027-5032. [PubMed: 4074675]

4. Fichtinger-Schepman AMJ, Van Oosterom AT, Lohman PHM, Berends F. cisDiamminedichloroplatinum (II)-induced DNA Adducts in Peripheral Leukocytes from Seven Cancer Patients : Quantitative Immunochemical Detection of the Adduct Induction and Removal after a Single Dose of cis-Diamminedichloroplatinum (II). Cancer Res. 1987; 47:3000-3004. [PubMed: 3552211]

5. Fichtinger-Schepman AMJ, Van Der Veer JL, Den Hartog JHJ, Lohman PHM. Adducts of the Antitumor Drug cis-Diamminedichloroplatinum (II) with DNA : Formation, Identification, and Quantitation. Biochemistry. 1985; 24:707-713. [PubMed: 4039603]

6. Fichtinger-Schepman AMJ, Van Der Velde-Visser SD, Van Dijk-Knijnenburg HCM, Van Oosterom AT, Baan RA, Berends F. Kinetics of the Formation and Removal of Cisplatin-DNA Adducts in Blood Cells and Tumor Tissue of Cancer Patients Receiving Chemotherapy : Comparison with in Vitro Adduct Formation. Cancer Res. 1990; 50:7887-7894. [PubMed: 2253228]

7. Plooy ACM, Fichtinger-Schepman AMJ, Schutte HH, van Dijk M, Lohman PHM. The quantitative detection of various Pt-DNA-adducts in Chinese hamster ovary cells treated with cisplatin: application of immunochemical techniques. Carcinogenesis. 1985; 6:561-566. [PubMed: 4039227]

8. Zou Y, Van Houten B, Farrell N. Sequence Specificity of DNA-DNA Interstrand Cross-Link Formation by Cisplatin and Dinuclear Platinum Complexes. Biochemistry. 2006; 33:5404-5410. [PubMed: 8180163]

9. Eastman A. The formation, isolation and characterization of DNA adducts produced by anticancer platinum complexes. Pharmacol Ther. 1987; 34:155-166. [PubMed: 3317449]

10. Rink SM, Hopkins PB. A Mechlorethamine-Induced DNA Interstrand Cross-Link Bends Duplex DNA. Biochemistry. 1995; 34:1439-1445. [PubMed: 7827092]

11. Rink SM, Hopkins PB. Direct evidence for dna intrastrand cross-linking by the nitrogen mustard mechlorethamine in synthetic oligonucleotides. Bioorg Med Chem Lett. 1995; 5:2845-2850.

12. Bizanek R, McGuinness BF, Nakanishi K, Tomasz M. Isolation and Structure of an Intrastrand Cross-Link Adduct of Mitomycin C and DNA. Biochemistry. 1992; 31:3084-3091. [PubMed: 1554696]

13. Rink SM, Lipman R, Alley SC, Hopkins PB, Tomasz M. Bending of DNA by the Mitomycin CInduced, GpG Intrastrand Cross-Link. Chem Res Toxicol. 1996; 9:382-389. [PubMed: 8839039] 
14. Iwamoto T, Hiraku Y, Olkawa S, Mizutani H, Kojima M, Kawanishi S. DNA intrastrand cross-link at the $5^{\prime}$-GA-3' sequence formed by busulfan and its role in the cytotoxic effect. Cancer Sci. 2004; 95:454-458. [PubMed: 15132775]

15. Masutani C, Kusumoto R, Yamada A. The XPV (xeroderma pigmentosum variant) gene encodes human DNA polymerase eta. Nature. 1999; 399:700-704. [PubMed: 10385124]

16. Lin Q, Clark AB, McCulloch SD, Yuan T, Bronson RT, Kunkel Ta, Kucherlapati R. Increased Susceptibility to UV-Induced Skin Carcinogenesis in Polymerase $\eta$-deficient Mice. Cancer Res. 2006; 66:87-94. [PubMed: 16397220]

17. Yao J, Dixon K, Carty MP. A Single (6-4) Photoproduct Inhibits Plasmid DNA Replication in Xeroderma Pigmentosum Variant Cell Extracts. Environ Mol Mutagen. 2001; 38:19-29. [PubMed: 11473384]

18. Yamada K, Takezawa J, Ezaki O. Translesion replication in cisplatin-treated xeroderma pigmentosum variant cells is also caffeine-sensitive: Features of the error-prone DNA polymerase(s) involved in UV-mutagenesis. DNA Repair (Amst). 2003; 2:909-924. [PubMed: 12893087]

19. Bassett E, King NM, Bryant MF, Hector S, Pendyala L, Chaney SG, Cordeiro-stone M. The Role of DNA Polymerase $\eta$ in Translesion Synthesis Past Platinum DNA Adducts in Human Fibroblasts The Role of DNA Polymerase $\eta$ in Translesion Synthesis Past Platinum-DNA Adducts in Human Fibroblasts. Cancer Res. 2004; 64:6469-6475. [PubMed: 15374956]

20. Chaney SG, Campbell SL, Bassett E, Wu Y. Recognition and processing of cisplatin- and oxaliplatin-DNA adducts. Crit Rev Oncol Hematol. 2005; 53:3-11. [PubMed: 15607931]

21. Albertella MR, Green CM, Lehmann AR, O'Connor MJ. A Role for Polymerase $\eta$ in the Cellular Tolerance to Cisplatin-Induced Damage. Cancer Res. 2005; 65:9799-9806. [PubMed: 16267001]

22. Box HC, Dawidzik JB, Budzinski EE. Free radical-induced double lesions in DNA. Free Radic Biol Med. 2001; 31:856-868. [PubMed: 11585704]

23. Raychaudhury P, Basu AK. Replication Past the $\gamma$-Radiation-Induced Guanine-Thymine CrossLink G[8,5-Me]T by Human and Yeast DNA Polymerase ๆ. J Nucleic Acids. 2010; 2010doi: 10.4061/2010/101495

24. Gu C, Wang Y. LC-MS/MS Identification and Yeast Polymerase $\eta$ Bypass of a Novel $\gamma$-IrradiationInduced Intrastrand Cross-Link Lesion G[8-5]C. Biochemistry. 2004; 43:6745-6750. [PubMed: 15157108]

25. Jiang Y, Hong H, Cao H, Wang Y. In Vivo Formation and In Vitro Replication of a GuanineThymine Intrastrand Cross-Link Lesion. Biochemistry. 2007; 46:12757-12763. [PubMed: 17929946]

26. Rydberg B. Nonenzymatic methylation of DNA by the intracellular methyl group donor Sadenosyl-L-methionine is a potentially mutagenic reaction. EMBO J. 1982; 1:211-216. [PubMed: 7188181]

27. Bishop RE, Pauly GT, Moschel RC. $O^{6}$-Ethylguanine and $O^{6}$-benzylguanine incorporated sitespecifically in codon 12 of the rat $\mathrm{H}$-ras gene induce semi-targeted as well as targeted mutations in Rat4 cells. 1996; 17:849-856.

28. Choi JY, Chowdhury G, Zang H, Angel KC, Vu CC, Peterson L, Guengerich FP. Translesion Synthesis across $O^{6}$-Alkylguanine DNA Adducts by Recombinant Human DNA Polymerases. J Biol Chem. 2006; 281:38244-38256. [PubMed: 17050527]

29. Pauly GT, Hughes SH, Moschel RC. Comparison of mutagenesis by $O^{6}$-methyl- and $O^{6}$. ethylguanine and $O^{4}$-methylthymine in Escherichia coli using double-stranded and gapped plasmids. Carcinogenesis. 1998; 19:457-461. [PubMed: 9525280]

30. Pauly GT, Moschel RC. Mutagenesis by $O^{6}$-Methyl-, $O^{6}$-Ethyl-, and $O^{6}$-Benzylguanine and $O^{4}$ Methylthymine in Human Cells: Effects of $O^{6}$-Alkylguanine-DNA Alkyltransferase and Mismatch Repair. Chem Res Toxicol. 2001; 14:894-900. [PubMed: 11453737]

31. Pauly GT, Hughes SH, Moschel RC. Mutagenesis in Escherichia coli by Three $O^{6}$-Substituted Guanines in Double-Stranded or Gapped Plasmids. Biochemistry. 1995; 34:8924-8930. [PubMed: 7612634]

32. Pauly GT, Hughes SH, Moschel RC. Response of Repair-Competent and Repair-Deficient Escherichia coli to Three $O^{6}$-Substituted Guanines and Involvement of Methyl-Directed Mismatch 
Repair in the Processing of $O^{6}$-Methylguanine Residues. Biochemistry. 1994; 33:9169-9177. [PubMed: 8049220]

33. Peterson LA, Hecht SS. $O^{6}$-Methylguanine Is a Critical Determinant of 4- (Methylnitrosamino)-1(3-pyridyl)-1-butanone Tumorigenesis in A/J Mouse Lung1. Cancer Res. 1991; 51:5557-5564. [PubMed: 1913675]

34. O'Flaherty DK, Wilds CJ. Synthesis, Characterization, and Repair of a Flexible $O^{6}-2^{\prime}$ Deoxyguanosine-alkylene- $O^{6}-2^{\prime}$-deoxyguanosine Intrastrand Cross-Link. Euro Chem J. 2015; 21:10522-10529.

35. Murata S, Mizumura Y, Hino K, Ueno Y, Ichikawa S, Matsuda A. Modular Bent DNAs: A New Class of Artificial DNAs with a Protein Binding Ability. J Am Chem Soc. 2007; 129:1030010301. [PubMed: 17672453]

36. Noll DM, Noronha aM, Miller PS. Synthesis and characterization of DNA duplexes containing an $N^{4}$ C-ethyl- $N^{4}$ C interstrand cross-link. J Am Chem Soc. 2001; 123:3405-3411. [PubMed: 11472110]

37. Patra A, Nagy LD, Zhang Q, Su Y, Müller L, Guengerich FP, Egli M. Kinetics, Structure, and Mechanism of 8-Oxo-7,8-dihydro-2'-deoxyguanosine Bypass by Human DNA Polymerase $\eta$. J Biol Chem. 2014; 289:16867-16882. [PubMed: 24759104]

38. Patra A, Zhang Q, Lei L, Su Y, Egli M, Guengerich FP. Structural and Kinetic Analysis of Nucleoside Triphosphate Incorporation Opposite an Abasic Site by Human Translesion DNA Polymerase $\eta$. J Biol Chem. 2015; 290:8028-8038. [PubMed: 25666608]

39. Boosalis MS, Petruska J, Goodman MF. DNA Polymerase Insertion Fidelity: gel assay for sitespecific kinetics. J Biol Chem. 1987; 262:14689-14696. [PubMed: 3667598]

40. O'Flaherty DK, Guengerich FP. Steady-State Kinetic Analysis of DNA Polymerase SingleNucleotide Incorporation Products. Curr Protoc Nucleic Acid Chem. 2014; 59:7.21.1-7.21.13. [PubMed: 25501593]

41. Chowdhury G, Guengerich FP. Liquid Chromatography-Mass Spectrometry Analysis of DNA Polymerase Reaction Products. Curr Protoc Nucleic Acid Chem. 2011; 7:7.16.1-7.16.11.

42. Christov PP, Angel KC, Guengerich FP, Rizzo CJ. Replication Past the $N^{5}$-MethylFormamidopyrimidine Lesion of Deoxyguanosine by DNA Polymerases and an Improved Procedure for Sequence Analysis of In Vitro Bypass Products by Mass Spectrometry. Chem Res Toxicol. 2009; 22:1086-1095. [PubMed: 19397282]

43. Zang H, Goodenough AK, Choi JY, Irimia A, Loukachevitch LV, Kozekov ID, Angel KC, Rizzo CJ, Egli M, Guengerich FP. DNA Adduct Bypass Polymerization by Sulfolobus solfataricus DNA polymerase Dpo4: Analysis and crystal structures of multiple base pair substitution and frameshift products with the adduct 1,N2-ethenoguanine. J Biol Chem. 2005; 280:29750-29764. [PubMed: 15965231]

44. Choi JY, Guengerich FP. Kinetic Evidence for Inefficient and Error-prone Bypass cross Bulky $N^{2}$ Guanine DNA Adducts by Human DNA Polymerase ı. J Biol Chem. 2006; 281:12315-12324. [PubMed: 16527824]

45. Vaisman A, Chaney SG. The Efficiency and Fidelity of Translesion Synthesis past Cisplatin and Oxaliplatin GpG Adducts by Human DNA Polymerase $\beta$. J Biol Chem. 2000; 275:13017-13025. [PubMed: 10777605]

46. Zhao Y, Biertumpfel C, Gregory MT, Hua YJ, Hanaoka F, Yang W. Structural basis of human DNA polymerase $\eta$-mediated chemoresistance to cisplatin. Proc Natl Acad Sci USA. 2012; 109:7269_ 7274. [PubMed: 22529383]

47. Mcculloch SD, Kokoska RJ, Masutani C, Iwai S, Hanaoka F, Kunkel TA. Preferential cis - syn thymine dimer bypass by DNA polymerase $\eta$ occurs with biased fidelity. Nature. 2004; 428:97100. [PubMed: 14999287]

48. Colis LC, Raychaudhury P, Basu AK. Mutational Specificity of $\gamma$-Radiation-Induced Guanine Thymine and Thymine - Guanine Intrastrand Cross-Links in Mammalian Cells and Translesion Synthesis Past the Guanine - Thymine Lesion by Human DNA Polymerase $\eta$. Biochemistry. 2008; 47:8070-8079. [PubMed: 18616294] 
49. Vaisman A, Masutani C, Hanaoka F, Chaney SG. Efficient Translesion Replication Past Oxaliplatin and Cisplatin GpG Adducts by Human DNA Polymerase $\eta$. Biochemistry. 2000; 39:4575-4580. [PubMed: 10769112]

50. Boiteux S, Laval J. Coding Properties of Poly(deoxycytidylic acid) Templates Containing Uracil or Apyrimidinic Sites: In Vitro Modulation of Mutagenesis by Deoxyribonucleic Acid Repair Enzyme. Biochemistry. 1982; 21:6746-6751. [PubMed: 6760893]

51. Sagher D, Strauss B. Insertion of Nucleotides Opposite Apurinic/Apyrimidinic Sites in Deoxyribonucleic Acid during in Vitro Synthesis : Uniqueness of Adenine Nucleotides. Biochemistry. 1983; 22:4518-4526. [PubMed: 6354260]

52. Schaaper RM, Glickman BW, Loeb LA. Mutagenesis resulting from depurination is an SOS process. Mut Res. 1982; 106:1-9. [PubMed: 6219285] 
A

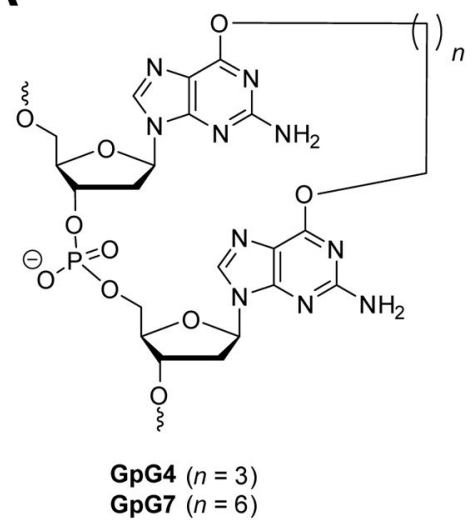

B

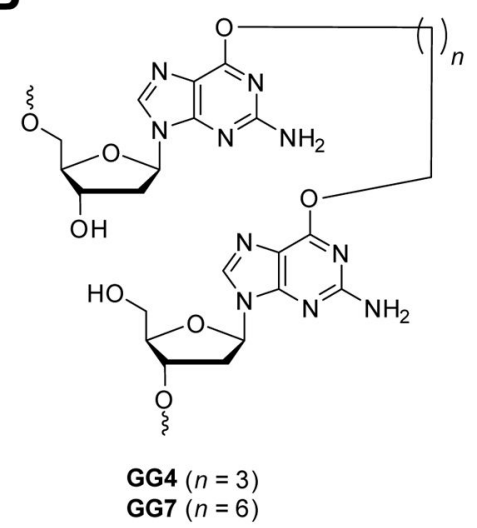

Figure 1.

Structures of the $O^{6}$-dG-alkylene- $O^{6}-\mathrm{dG}$ IaCL containing $(\mathbf{A})$ and lacking $(\mathbf{B})$ the phosphodiester linkage between the $O^{6}$-linked nucleotides. 


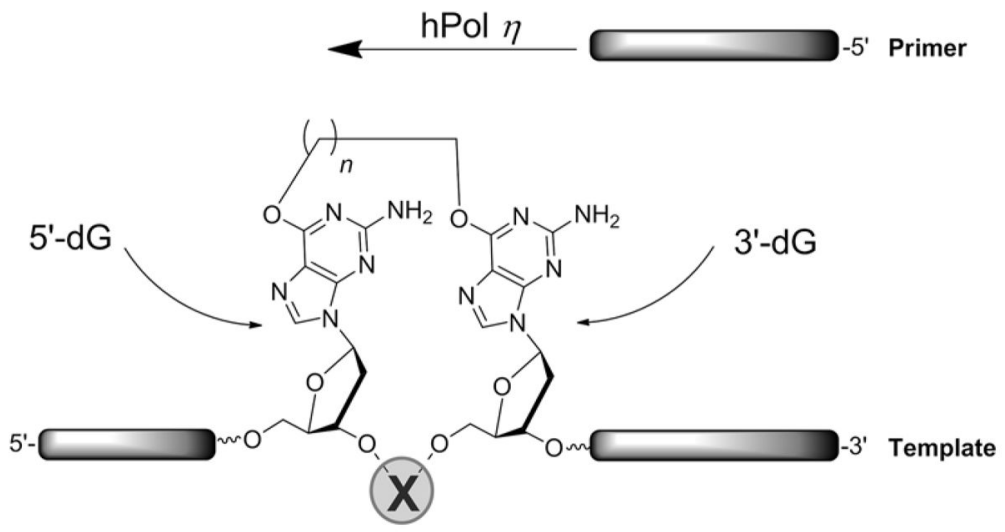

Figure 2.

Pictorial representation of primer extension assay using hPol $\eta$, where $\mathbf{X}$ represents the presence, or lack, of a phosphodiester linkage. Frameshift adduct formation $(-1)$, observed primarily at the $5^{\prime}$-dG residue for GG4 and GG7, are characterized by hPol $\eta$ skipping the template nucleotide. 

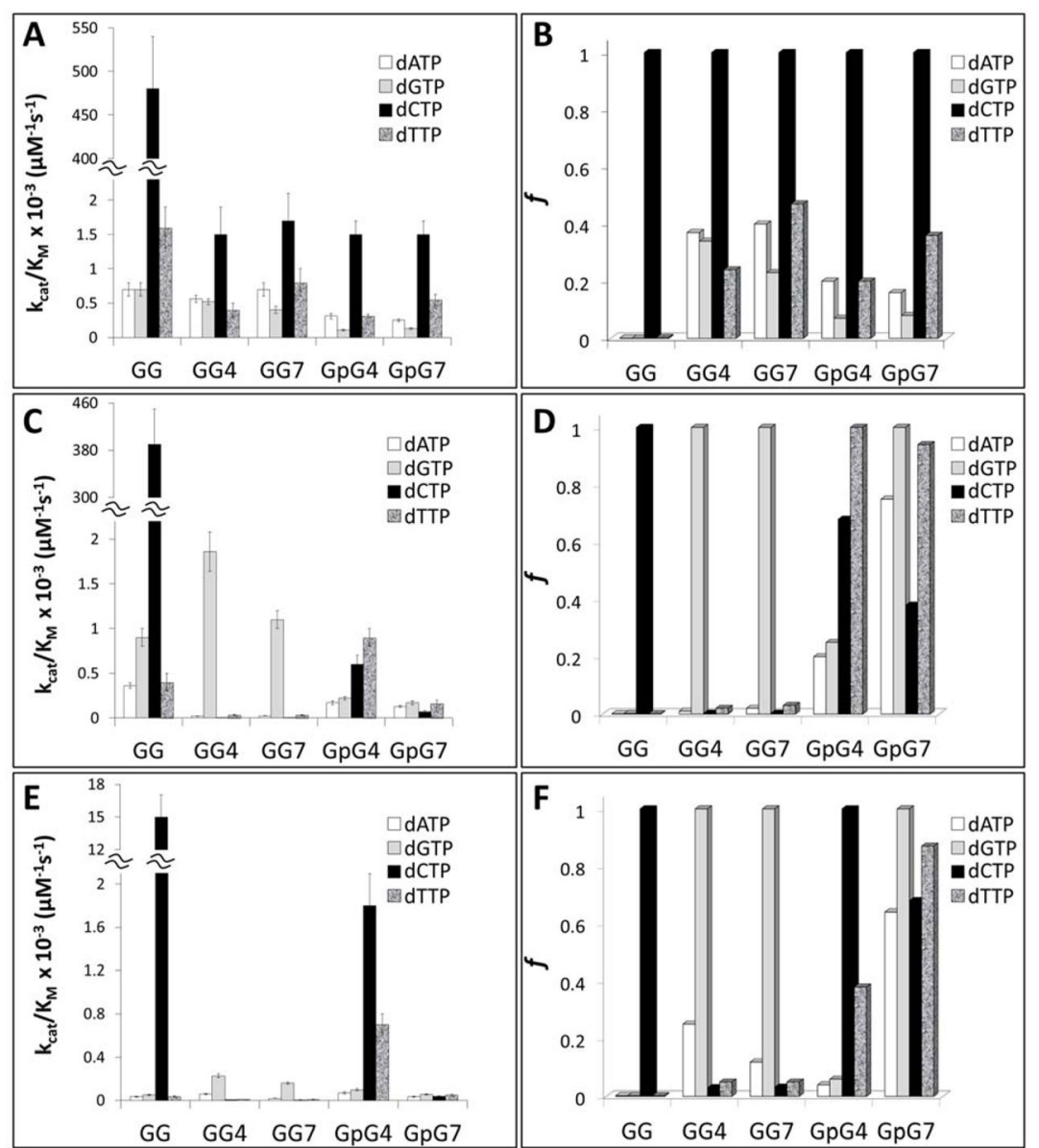

Figure 3.

Summary of steady-state kinetics of incorporation of dNTP opposite IaCL-containing template GG4, GG7, GpG4, GpG7 (5'-AC $\underline{\mathbf{X X}}$ CT CAC ACT) and unmodified template (GG) by hPol $\eta$. DNA primer sequence identity were $3^{\prime}$-GA GUG TGA T(FAM)-5' (Panel

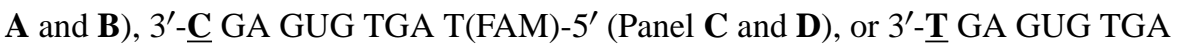
T(FAM)-5' (Panel E and F). Tabulated values are reported in Supporting Information. 

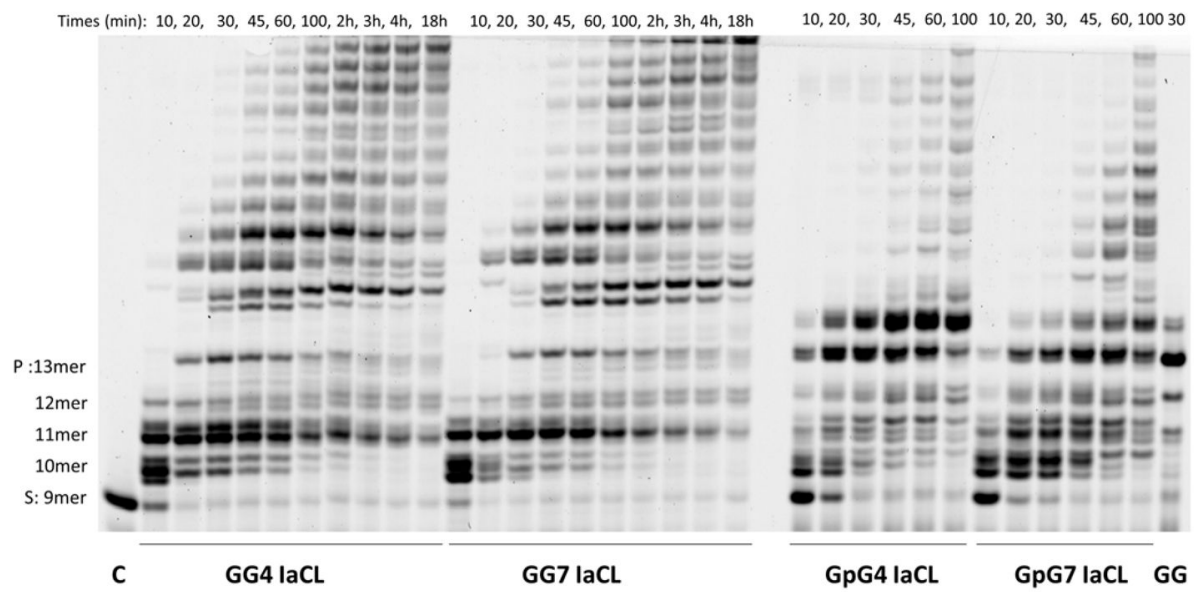

Figure 4.

Time course assay of primer full extension ( $25 \mu \mathrm{M}$ annealed DNA) by hPol $\eta(3.4 \mu \mathrm{M}$ for damaged and $1 \mu \mathrm{M}$ undamaged DNA) in presence of all four dNTPs ( $1 \mathrm{mM}$ mixture). DNA duplexes were composed of IaCL-containing template GG4, GG7, GpG4, GpG7 (5'-AC

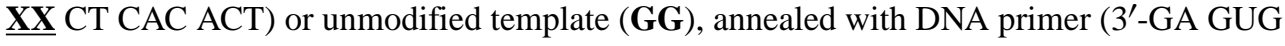
TGA T(FAM)-5' . DNA template used is indicated at the bottom, product length identified on the left-hand side, reaction times are indicated at the top (in minutes unless stated otherwise). Note that only the $30 \mathrm{~min}$ reaction time is shown for the undamaged control (GG) (data for other time points not shown). Extended products were resolved by $17 \%$ denaturing PAGE (19:1) and visualized via fluorography. 

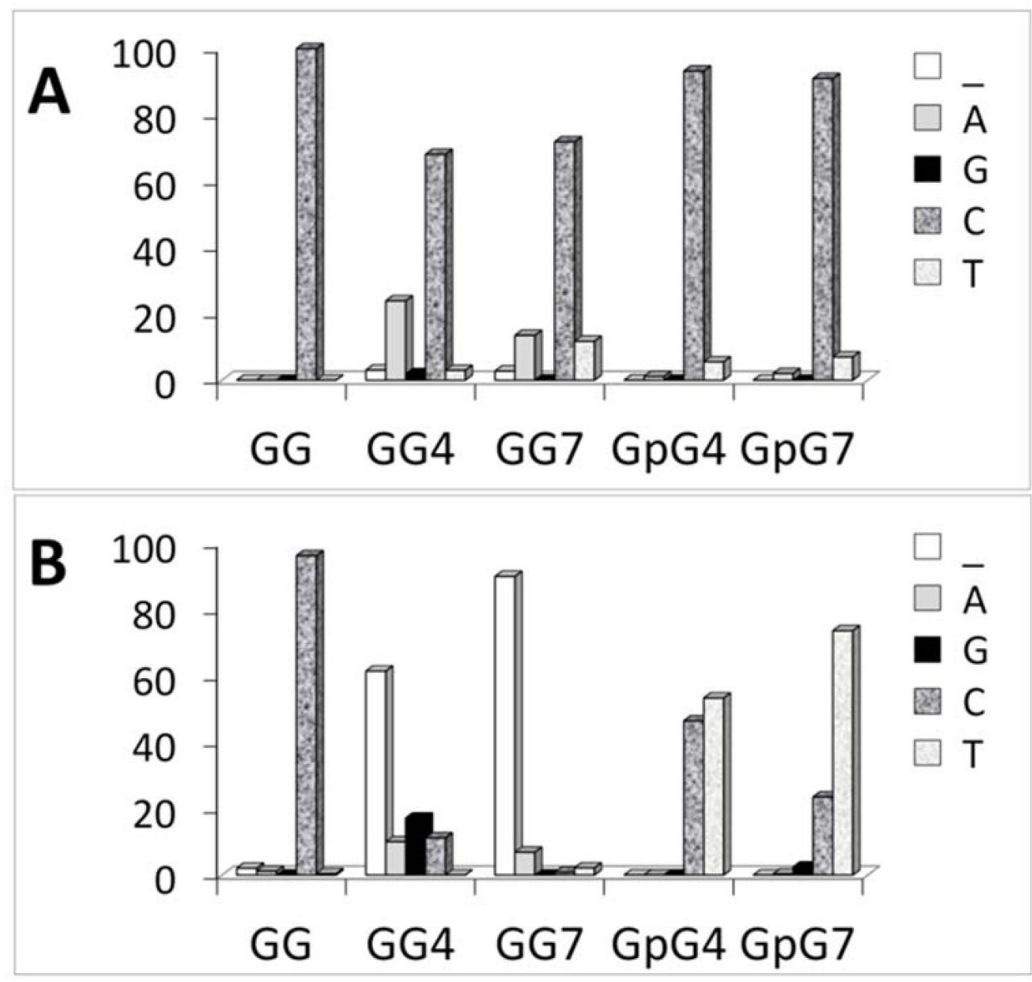

Figure 5.

Insertion profiles by hPol $\eta$ opposite the (A) $3^{\prime}-\mathrm{dG}$ and the (B) $5^{\prime}$-dG of the $O^{6}$-dG-alkylene$O^{6}$-dG IaCL and unmodified control. DNA sequence contexts were as follows: IaCLcontaining template (GG4, GG7, GpG4, GpG7 or unmodified template GG) had sequence

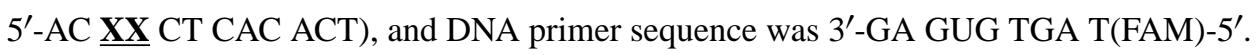
Tabulated LC-MS extension products and percentages can be found in Supporting Information. 